\title{
Flexibilização de avaliações acerca de profissões após um programa em orientação profissional ${ }^{1}$
}

\author{
Nathália Sabaine Cippola ${ }^{2}$ \\ Camila Domeniconi \\ Universidade Federal de São Carlos, São Carlos-SP, Brasil \\ Andréia Schmidt \\ Universidade de São Paulo, Ribeirão Preto-SP, Brasil
}

\section{Resumo}

A forma como são avaliadas algumas profissões pode interferir na escolha profissional de jovens. Este estudo teve como objetivo verificar os efeitos de um processo de orientação profissional (OP) sobre a avaliação de algumas afirmações sobre profissões em seis estudantes do segundo ano do ensino médio considerados flexíveis e inflexíveis. Antes e após o programa de OP foram aplicadas a Escala de Afirmações Profissionais e a Escala de Rigidez. Observou-se que após a OP houve mudanças nas avaliações das afirmações entre participantes considerados flexíveis e inflexíveis; algumas afirmações foram mais fáceis de serem mudadas que outras. O procedimento mostrou-se eficaz quanto às mudanças nas avaliações de afirmações, e o repertório anterior dos indivíduos pareceu influenciar na flexibilização das avaliações.

Palavras-chave: análise do comportamento, orientação vocacional, flexibilização

\section{Abstract: Flexibility of assessments about professions following a program in career guidance}

The way some professions are assessed may interfere with the professional choice of young people. This study aimed to verify the effects of a process of professional orientation (PO) on the evaluation of some statements about professions in six high school sophomores considered flexible and inflexible. Before and after the PO program, the Professional Affirmations Scale and the Rigidity Scale were applied. It was observed that after the PO there were changes in the assessments of the affirmations between participants considered flexible and inflexible; some statements were easier to change than others. The procedure proved to be effective for changes in assay evaluations, and the individuals' previous repertoire seemed to influence the flexibilization of assessments. Keywords: behavioral analysis, vocational orientation, flexibilization

Resumen: Flexibilización de las evaluaciones sobre las profesiones después de un programa de orientación profesional La manera como se valoran las profesiones puede interferir en la elección profesional de los jóvenes. Esta investigación tuvo como objetivo verificar los efectos de un proceso de orientación profesional (OP) sobre la valoración de algunas afirmaciones sobre profesiones por seis estudiantes del segundo año de la enseñanza media considerados flexibles e inflexibles. Antes y después del programa de OP fueran utilizadas la Escala de Afirmaciones Profesionales y la Escala de Rigidez. Se constató que luego de la OP hubo cambios en las valoraciones de las afirmaciones entre participantes considerados flexibles e inflexibles; también se verificó que algunas afirmaciones fueron más fáciles de transformar que otras. El procedimiento evidenció ser eficaz para los cambios en las valoraciones de afirmaciones y el repertorio precedente de los sujetos pareció determinar en la flexibilización de las mismas.

Palabras clave: análisis del comportamiento, orientación vocacional, flexibilización

\footnotetext{
${ }^{1} \mathrm{O}$ presente trabalho faz parte do terceiro estudo da tese de doutorado da aluna Nathália Sabaine Cippola, com orientação de Camila Domeniconi e co-orientação de Andreia Schmidt. O trabalho foi financiado pela Coordenação de Aperfeiçoamento de Pessoal de Nível Superior - CAPES/Brasil.

${ }^{2}$ Endereço para correspondência: Rodovia Washington Luís, Km 235, Caixa Postal 676, 13565-905, São Carlos, SP.

E-mail: nathycippola@hotmail.com
} 
O processo de orientação profissional tem como foco trabalhar junto ao orientando para que ele possa tomar decisões sobre seu futuro profissional com mais informações sobre seus interesses e habilidades (Moura, 2011). A orientação profissional atua nos processos de autorreflexão, clarificação de valores e perspectivas pessoais e autoconceito, levando o indivíduo a refletir sobre si mesmo, conhecer-se melhor, descobrir quais valores, interesses, exploração de profissões, motivações e potencialidades podem ser desenvolvidas (Ribeiro \& Uvaldo, 2007).

Estudos realizados com base no marco teórico da Análise do Comportamento também têm sido realizados com o objetivo de investigar os fenômenos relacionados ao processo de escolha e tomada de decisão dos indivíduos acerca do exercício profissional. Um dos primeiros trabalhos a apresentar alguma referência sobre a aplicação dos princípios da Análise do Comportamento em orientação profissional foi realizado por Martins (1978), que trouxe explicações a respeito do "comportamento vocacional".

Sob o enfoque dessa abordagem, outros trabalhos investigaram as variáveis envolvidas no momento de uma escolha profissional (Krumboltz, 1992, 1998; Krumboltz \& Worthington, 1999; Mitchell \& Krumboltz, 1996; Mitchell, Levin, \& Krumboltz, 1999; Moura, Sampaio, Paranzini, Menezes, \& Rodrigues, 2003; Lent, Brown, \& Hackett, 2000; Swanson \& Gore, 2000) e no desenvolvimento de técnicas no processo de orientação profissional (Sharf, 1992). Foram investigados ainda os modelos de aprendizagem social baseados na proposta de Bandura (1979) (Sampson Jr, Peterson, Lenz, \& Reardon, 1992) e realizados trabalhos que avaliaram programas em orientação profissional (Moura, Sampaio, Gemelli, Rodrigues, \& Menezes, 2005; Moura \& Menezes, 2004), e incluíram relatos de experiências nos atendimentos (Moura \& Silveira, 2002).

O comportamento de escolha é multideterminado por variáveis de diferentes naturezas, entre elas aquelas relativas à espécie, à história de vida do sujeito e à cultura na qual ele se insere (Skinner, 1969). As variáveis inatas referem-se à hereditariedade e podem favorecer ou dificultar o desempenho de algumas atividades (e.g., estatura, acuidade visual, estrutura física etc.). As variáveis relacionadas à aprendizagem ao longo da vida do sujeito dizem respeito às informações, experiências, aos modelos e relacionamentos que um indivíduo estabelece durante a vida com pais, parentes, professores, colegas, namorados e outros). A variável cultural inclui processos de aprendizagem de valores sociais e conceitos culturais a respeito das atividades profissionais em certa comunidade como, por exemplo, modismo das profissões, status social, diferenças de gênero, remuneração, entre outras. Cabe ao orientador e ao orientando engajarem-se em diversos comportamentos relacionados ao levantamento dessas variáveis e relacioná-las, tanto às habilidades pessoais, quanto às questões de escolha profissional, com o objetivo específico de aumentar a probabilidade de que o comportamento de escolha ocorra (Moura \& Menezes, 2004).

Os comportamentos de escolher e tomar decisão podem ser ensinados e aprendidos e, de acordo com Skinner (1989), aprender a tomar uma decisão é identificar as variáveis que interferem nessa decisão e analisar as possíveis consequências de escolher cada uma das alternativas disponíveis. Um exemplo de situação que demanda uma escolha no processo de decisão profissional é cursar uma universidade na cidade onde reside ou fora dela. $\mathrm{O}$ indivíduo precisa analisar o que possivelmente irá acontecer nas duas condições: quais seriam as facilidades em morar com os pais e quais seriam as dificuldades, o que poderia aprender longe de casa ou estando em casa, em qual das duas universidades seria possível atingir seus objetivos de forma satisfatória, qual seu repertório de autonomia para morar em outra cidade etc. Ao analisar essas e outras variáveis o orientando deveria escolher qual curso lhe garantiria maior satisfação, menor prejuízo e quais consequências gostaria (ou seria capaz) de enfrentar.

A decisão profissional envolve algumas contingências culturalmente difundidas e já pré-estabelecidas e que podem dificultar o processo de escolha como, por exemplo, a posição socioeconômica da família, o status que a profissão oferece, a contradição entre o conteúdo e a forma da profissão, a distorção à respeito da realidade profissional (ênfase nos pontos positivos ou negativos da profissão), a crença de que o mercado de trabalho será o mesmo quando o curso terminar, a divergência entre a preparação profissional e a realidade profissional, a relação entre matéria e profissão, a relação entre sexo e profissão, influências diretas ou veladas sobre os valores dos pais a respeito de determinadas profissões, profissões tradicionais nas famílias, entre outras (Almeida, Melo- Silva, \& Santos, 2017; Leal, Melo- Silva, \&Teixeira, 2015; Levenfus \& Soares, 2002; Moraes \& Lima, 2014; Oliveira \& Dias, 2013; Sawitri, Creed, \& Zimmer-Gemback, 2014).

Os orientandos podem fazer escolhas apenas com base nas contingências culturalmente difundidas e não naquelas que são produzidas pelo seu comportamento ou que se referem às suas habilidades e interesses. Por exemplo, o indivíduo pode avaliar a escolha de uma profissão mediante uma regra social em relação à remuneração dessa profissão ou se mais homens ou mulheres exercem aquela atividade. Isso pode ser ruim em um processo de decisão, pois a sensibilidade do comportamento humano diante das consequências produzidas por ele 
garante que os comportamentos se modifiquem quando as contingências mudarem (Catania, 1999). É provável que adolescentes que avaliam várias alternativas diferentes de descrições sobre contingências de escolha profissional sejam mais aptos a escolher, uma vez que um indivíduo menos sensível à sua história, suas habilidades e interesses, e mais rígido quanto ao seguir regras socialmente difundidas, pode ficar menos satisfeito futuramente com sua escolha profissional.

Alguns estudos apontam que a principal fonte de variabilidade comportamental está na capacidade de os organismos ficarem mais sensíveis às contingências naturais e de seguir menos as instruções e regras socialmente difundidas (Albuquerque, 2001, 2005; Albuquerque, Paracampo, \& Allan, 2017; Albuquerque, Silva, \& Paracampo, 2014; Albuquerque, Souza, Matos, \& Paracampo, 2003; Albuquerque \& Paracampo 2010; Albuquerque, Reis, \& Paracampo, 2006; Albuquerque \& Silva, 2006; Catania, 1998; Catania, Matthews, \& Shimoff, 1990; Catania, Shimoff, \& Matthews, 1989; Cerutti, 1989; Joyce \& Chase, 1990; LeFrancois, Chase, \& Joyce, 1988; Lima, Albuquerque, \& Paracampo, 2017; Paracampo \& Albuquerque, 2004; Paracampo, et al., 2001; Paracampo, Souza, \& Albuquerque, 2014; Santos, Albuquerque, \& Paracampo, 2004; Skinner, 1969; Torgrud \& Holborn, 1990; Zettle \& Hayes, 1982). Esses estudos sugerem que o seguimento estrito de instruções pode levar os indivíduos a comportamentos muito rígidos e a dificuldades de adaptar seus comportamentos a situações novas, para as quais não há desempenhos pré-estabelecidos. Nesse sentido, algumas variáveis da dimensão rigidez-flexibilidade podem influenciar o comportamento do indivíduo de tomar decisões.

Alguns estudos (Rehfisch, 1958; Kravas, 1973; Naftulin, Donnelly, \&Wolkon, 1974; Linn, Moravec, \& Zeppa, 1982; Vollhardt, 1990; Schaie, Dutta, \& Willis, 1991; Oliveira \& Albuquerque, 2007; Paracampo, Souza, Matos, \& Albuquerque, 2001) testaram a dimensão flexibilidade-inflexibilidade em situações experimentais para observar padrões de resposta. Para isso utilizaram o questionário desenvolvido por Rehfisch (1958), que contém 39 afirmações que os participantes devem avaliar como verdadeiras e falsas. A racional do uso dessa escala é que indivíduos com uma forte história de seguimento de regras ao longo da vida podem ser avaliados como mais rígidos e, com isso, tenderem a seguir mais instruções, tanto em situações de laboratório, como em ambiente natural. Os resultados da escala parecem ser uma tendência permanente, pois esta avalia a história do indivíduo que reflete na rigidez e que pouco muda ao longo da vida (Rehfisch, 1958).
A dimensão flexibilidade-rigidez pode interferir em diferentes aspectos da vida dos indivíduos, incluindo a escolha profissional. A OP, nesse sentido, pode ser importante como processo que promove melhoria na capacidade de discriminação e análise das variáveis envolvidas no processo de tomada de decisão, inclusive das regras socialmente difundidas implicadas neste processo. Durante a tomada de decisão, o orientador apresenta as variáveis implicadas na tomada de decisão por uma profissão ou outra, permitindo ao indivíduo uma melhor descrição das situações e das consequências prováveis para cada escolha, permitindo a possibilidade de analisar variáveis além das que o ambiente imediato dele proporciona (Oliveira, Silva, Garcia, Melo-Silva, \& Teixeira, 2014; Ribeiro, 2011; Silva, Oliveira, \& Melo-Silva, 2014).

Muitas propostas de atendimento em OP demonstram eficácia (Melo-Silva, 2011; Manaia, Leal, Shimada, \& Melo-Silva, 2014; Melo-Silva \& Alonso, 2013; Teixeira, Lassance, \& Melo-Silva, 2012), inclusive aquelas baseadas nos pressupostos teóricos da Análise do Comportamento (Moura et al., 2005; Moura \& Menezes, 2004; Moura \& Silveira, 2002), mas há uma lacuna na literatura a respeito da relação entre a participação em programas de OP e a flexibilização de avaliações acerca de profissões e o impacto das regras socialmente carregadas de significado sobre as escolhas profissionais. Também não foram encontrados na literatura estudos que utilizaram a dimensão flexibilidade-inflexibilidade em relação à escolha profissional.

Considerando que o processo de OP pode ter como um dos seus efeitos levar o orientando a melhorar descrições de contingências relacionadas à escolha profissional, e que essas escolhas podem estabelecer contingências futuras que sejam satisfatórias ao indivíduo, parece pertinente aumentar o contingente de investigações direcionadas para esta temática. Por isso, o presente trabalho teve como objetivo verificar os efeitos de um processo de orientação profissional sobre a avaliação de descrições de contingências ligadas às profissões e à escolha profissional culturalmente difundidas (eventuais mudanças nas avaliações) por estudantes de ensino médio considerados flexíveis e inflexíveis.

\section{Método}

\section{Participantes}

Participaram da pesquisa seis estudantes do segundo ano do ensino médio (quatro do sexo feminino e dois do sexo masculino), com idade entre 15 e 16 anos e que pretendiam prestar vestibular. Os participantes 
não haviam participado de outras pesquisas relacionadas à Psicologia e não tiveram experiência prévia com Orientação Profissional. Os mesmos foram selecionados a partir de resultados de um pré-teste (Escala de Afirmações Profissionais), que compreendia uma avaliação de 40 afirmações sobre profissões, vestibular e escolha profissional, desenvolvido pelas pesquisadoras a partir de uma entrevista com alguns psicólogos especializados em OP. Os estudantes avaliaram essas afirmações como verdadeiras ou falsas em uma escala de sete pontos, variando de $\mathrm{V}$ a F. Ao todo 216 participantes do segundo ano do ensino médio responderam ao questionário. Destes, foram selecionados os seis estudantes que consideraram as mesmas frases como "completamente falsas" e "completamente verdadeiras". O procedimento teve início mediante assinatura do Termo de Consentimento Livre e Esclarecido pelos pais e Termo de Assentimento dos adolescentes, conforme orientação do Comitê de Ética em Pesquisa com Seres Humanos, CAE: 35155914.1.0000.5504.

\section{Local}

As sessões experimentais foram realizadas na escola onde os participantes estudavam, durante o período escolar. Parte do procedimento de coleta ocorreu em uma sala de aula, que continha cerca de 50 carteiras escolares, sendo que seis delas foram posicionadas em formato de $\mathrm{U}$, de modo que todos os participantes pudessem observar uns aos outros. Os procedimentos realizados com auxílio de computadores foram realizados na sala de informática da escola, que continha 24 máquinas, com monitores, teclados e mouse individuais. A sala estava organizada em quatro bancadas enfileiradas horizontalmente, com seis computadores em cada uma delas.

\section{Situação e materiais}

Os estudantes passaram por um procedimento de pré e pós-avaliação com tarefas de emparelhamento com o modelo (MTS) que testavam se os participantes relacionavam um conjunto de afirmações sobre escolha profissional e profissões (aqui denominado conjunto $\mathrm{C}$ ver adiante) a símbolos de "correto", "falso" e "neutro" (denominado conjunto A). Para fins de descrição dessas avaliações, daqui em diante elas serão chamadas de testes (pré ou pós) AC/CA.

Os estímulos visuais do conjunto $\mathrm{C}$, empregados no procedimento de pré e pós-avaliação, foram seis afirmações escritas, retiradas da Escala de Afirmações Profissionais, sendo três delas descritores de contingências consideradas previamente verdadeiras pelos participantes (C1): "Sou livre para escolher o que quiser" (C1.1); "Dinheiro é o mais importante na profissão" (C1.2); "Apenas mulheres trabalham com profissões relacionadas às crianças" (C1.3), e outras três, descritoras de contingências consideradas previamente falsas pelos participantes (C2): "Devo analisar meus pontos fracos também na hora da escolha profissional" (C2.1); "Devo considerar vários aspectos para escolher minha profissão" (C2.2); "Verificar o mercado de trabalho é fundamental para a escolha do profissional" (C2.3). O conjunto A foi composto por duas imagens: uma imagem em verde com sinal de "correto" (A1) e um sinal em vermelho simbolizando "errado" (A2). Além disso, foi utilizado um sinal com suposto significado neutro, representado por uma mão com o polegar apontado para a horizontal como um terceiro estímulo de comparação. A representação dos estímulos pode ser observada na Figura 1. Colunas ilustram os conjuntos de estímulos e linhas ilustram grupos de estímulos de cada conjunto, mais o estímulo neutro.

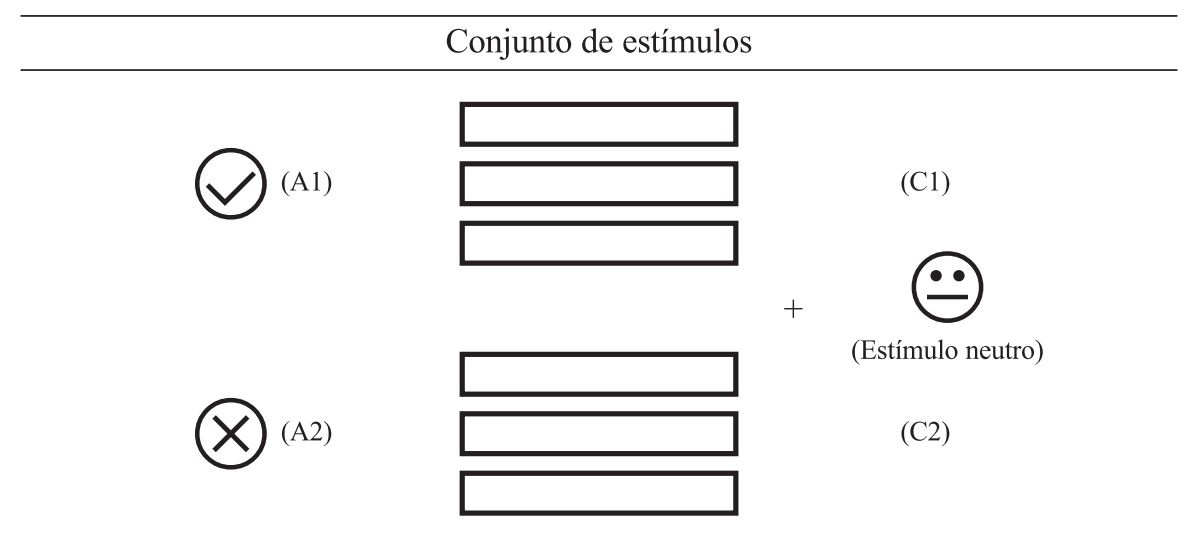

Figura 1. Estímulos que empregados nos testes AC/CA e sua alocação nos conjuntos 
Os estímulos visuais funcionavam, ora como modelo, ora como comparação, dependendo do tipo de teste. Por exemplo, nos testes CA, uma das seis afirmações aparecia escrita no centro da tela do computador (estímulo modelo) e, após o participante clicar sobre ela (resposta de observação), os estímulos do conjunto A, mais o estímulo neutro, eram apresentados na parte inferior da tela, lado a lado. Em todas as tentativas o estímulo modelo era apresentado e, após um a resposta de observação, eram apresentados os estímulos-comparação.

$\mathrm{O}$ gerenciamento de procedimentos e o armazenamento de dados foi feito por um sistema Web, LECH-GEIC (Gerenciador de Ensino Individualizado por Computador), desenvolvido por Orlando (2009), que viabilizou a autoria e a aplicação remota do procedimento. O sistema de funcionamento online foi executado em microcomputadores.

Entre a pré e pós-avaliação (testes $\mathrm{AC} / \mathrm{CA}$ ), os estudantes participaram de um programa de Orientação Profissional sob o enfoque da Análise do Comportamento. O programa contou com nove sessões, e materiais como folhas sulfite, caneta, lápis e borracha foram utilizados em todas as sessões.

Para a caracterização inicial de repertório dos participantes foram utilizados os seguintes instrumentos:

Escala de Rigidez: questionário elaborado por Rehfisch (1958) baseado no Minnesota Multiphasic Personality Inventory (MMPI) e no California Personality Inventory. A Escala de Rigidez contém 39 itens do tipo verdadeiro/falso e tem o objetivo de observar um padrão de comportamento mais ou menos rígido dos indivíduos. A partir do gabarito é feita a soma de respostas corretas e incorretas. São considerados como flexíveis os indivíduos com acertos iguais ou inferiores a $25 \%$ (11 acertos ou menos) e inflexíveis aqueles com pontuação igual ou acima de $75 \%$ ( 29 acertos ou mais). Neste estudo, foi utilizada a tradução em português feita por Jonas (2001).

Escala de Afirmações Profissionais: foi utilizado um questionário elaborado pelas pesquisadoras a partir da consulta com quatro profissionais de psicologia que trabalham com orientação profissional. O contato feito com os profissionais ocorreu por telefone e foi marcada uma entrevista com duração de, aproximadamente, 50 minutos. A pesquisadora explicou os objetivos da pesquisa, e os profissionais mencionaram algumas das afirmações que mais observavam em contexto clínico em procedimento de orientação profissional e que descreviam contingências verdadeiras e falsas a respeito das profissões, vestibular e tomada de decisão profissional. O critério para escolha das afirmações era a descrição de contingências que descreviam antecedentes, comportamentos e consequências que apareciam com frequência no discurso de candidatos à orientação profissional e que os profissionais consideravam dificultar a tomada de decisão profissional dos jovens em função de serem afirmações socialmente carregadas de significado.

\section{Procedimento}

A coleta de dados foi constituída por cinco fases: caracterização inicial do repertório, pré-teste, programa em orientação profissional, pós-teste e avaliação final. A Tabela 1 apresenta todas as fases, procedimentos e instrumentos utilizados.

Fase 1. Caracterização do repertório. Os participantes responderam à Escala de Afirmações Profissionais instruídos pela pesquisadora sobre como utilizá-la: "Quanto mais perto da palavra você colocar o ' $\mathrm{x}$ ', mais você considera esta afirmação como verdadeira/falsa". As seis afirmações que foram avaliadas pelos participantes nos extremos da escala (como "mais verdadeiras" ou "mais falsas") foram utilizadas nos procedimentos posteriores. Além disso, esses participantes, ainda na caracterização inicial do repertório, preencheram a Escala de Rigidez (Rehfisch, 1958).

Fase 2. Pré-teste das relações $A C / C A$. Esta fase teve como objetivo verificar as relações feitas pelos participantes entre as afirmações levantadas na Escala de Afirmações Profissionais (estímulos do conjunto C) e os símbolos significativos verdadeiro/falso (conjunto A), antes de qualquer ensino. A forma como os participantes avaliavam as afirmações (se corretas/verdadeiras ou se incorretas/falsas) não era consequenciada pela pesquisadora, ou seja, não era fornecido qualquer feedback para o participante sobre a forma como ele avaliava as afirmações. Porém, se algum participante avaliasse as afirmações de forma diferente da forma como avaliou a mesma afirmação na Fase 1 (Escala de Afirmações Profissionais), considerando alguma afirmação do conjunto $\mathrm{C} 1$ como falsa ou alguma afirmação do conjunto $\mathrm{C} 2$ como verdadeira, ele seguiria com o procedimento, mas seus dados seriam descartados da análise. Esse cuidado foi planejado em função do objetivo do estudo: ao se buscar verificar se o processo de OP poderia alterar a forma como o participante avaliava certas afirmações socialmente difundidas sobre profissões ou sobre a escolha profissional, era importante que esses participantes mantivessem, nesse momento, a mesma avaliação sobre as afirmações nos dois momentos.

Nessa fase cada tentativa no teste CA consistia na apresentação de um estímulo do conjunto $\mathrm{C}$ (C1.1, C1.2, C1.3, C2.1, C2.2, C2.3) como modelo, e os estímulos do conjunto A como comparações, mais o símbolo neutro, totalizando sempre três comparações por tentativa. 
Na sessão de pré-teste da relação CA foram apresentadas 36 tentativas, sendo apresentadas 18 tentativas cujos modelos eram as afirmações $\mathrm{C} 1$ e 18 tentativas em que o modelo eram afirmações $\mathrm{C} 2$, totalizando três apresentações de cada um dos seis estímulos-modelos pertencentes ao conjunto C. Cada participante passou por uma única sessão CA.

$\mathrm{O}$ pré-teste da relação $\mathrm{AC}$ foi realizado com o mesmo objetivo e a mesma estrutura do pré-teste CA, com uma única diferença: os estímulos de comparação para as tentativas eram uma das três afirmações corretas, uma das três afirmações falsas mais o estímulo neutro. Cada arranjo foi testado duas vezes com um dos modelos verdadeiros do conjunto $\mathrm{C} 1$ (C1.1, C1.2 e C1.3) e duas vezes com cada uma dos modelos falsos do conjunto $\mathrm{C} 2$ (C2.1, C2.2. e C2.3), totalizando 18 tentativas cada e 36 no total. Tanto no teste $\mathrm{AC}$ quanto no teste $\mathrm{CA}$ a tentativa iniciava com a apresentação do estímulo-modelo, seguida pela resposta de observação e a escolha do estímulo de comparação que o participante julgava estar melhor relacionado com o modelo; após a escolha do comparação, todos os estímulos desapareciam da tela e três segundo depois um novo modelo era apresentado.

Tabela 1

Descrição das fases do procedimento, com objetivo e instrumentos utilizados

\begin{tabular}{lll}
\hline \multicolumn{1}{c}{ Fases } & \multicolumn{1}{c}{ Objetivo } & \multicolumn{1}{c}{ Instrumento } \\
\hline 1. Caracterização do repertório & $\begin{array}{l}\text { Avaliar o repertório de entrada } \\
\text { dos participantes em relação à } \\
\text { avaliação das afirmações e rigidez }\end{array}$ & $\begin{array}{l}\text { Aplicação da Escala de } \\
\text { Afirmações profissionais } \\
\text { e da Escala de Rigidez }\end{array}$ \\
\hline 2. Pré-teste & $\begin{array}{l}\text { Avaliar desempenho } \\
\text { em Pré-teste da relação } \\
\text { C-A e A-C }\end{array}$ & $\begin{array}{l}\text { MTS } \\
\text { Implentativas C-A }\end{array}$ \\
& $\begin{array}{l}\text { Implentar um programa baseado } \\
\text { na abordagem da Análise do } \\
\text { comportamento estruturado em }\end{array}$ & $\begin{array}{l}\text { Realizar os procedimentos } \\
\text { em grupo em nove sessões } \\
\text { de orientação profissional }\end{array}$ \\
& $\begin{array}{l}\text { quatro eixos: autoconhecimento, } \\
\text { informação profissional, projeto } \\
\text { de vida e domada de decisão }\end{array}$ & \\
\hline 4. Pós-teste & $\begin{array}{l}\text { Avaliar desempenho em } \\
\text { Pós-teste da: Relação A-C/ C-A }\end{array}$ & $\begin{array}{l}\text { MTS } \\
\text { 3rama em Orientação Profissionativas C-A }\end{array}$ \\
& $\begin{array}{l}\text { Avaliar o repertório após } \\
\text { procedimento dos participantes em } \\
\text { relação a avaliação das afirmações }\end{array}$ & Aplicação da Escala de \\
& Afirmações profissionais \\
\hline 5. Avaliação Final & & \\
\hline
\end{tabular}

Fase 3. Programa em orientação profissional. O programa foi composto por nove encontros, que ocorriam duas vezes na semana, com a duração de uma hora e quinze minutos cada. O programa foi baseado no estudo de Moura e Silveira (2002) e seguiu quatro eixos:

$1^{\circ}$ Eixo - Autoconhecimento: esta etapa consistiu na promoção da discriminação das variáveis pessoais relacionadas às dificuldades de decisão por meio do autoconhecimento (identificação de habilidades, interesses, potenciais, valores, expectativas etc.).

$2^{\circ}$ Eixo - Conhecimento da realidade profissional: foi promovido o aumento da discriminação das variáveis profissionais (opções profissionais, cursos preparatórios, áreas de atuação, mercado de trabalho) e por informações de várias fontes (manuais, revistas, vídeos e entrevistas com profissionais).

$3^{\circ}$ Eixo - Projeto profissional e projeto de vida: foram realizadas atividades em que o orientando pudesse se projetar em um tempo futuro e conseguisse organizar objetivos profissionais (curso, local de trabalho, pós-graduação etc.) e objetivos pessoais (metas pessoais, familiares).

$4^{\circ}$ Eixo - Tomada de decisão: nesta etapa foi discutida a tomada de decisão pela análise dos critérios de exclusão/ inclusão de opções de cada orientando e a consideração de consequências reforçadoras a médio e longo prazo para cada opção.

Fase 4. Pós-teste das relações AC/CA. Para verificar os efeitos do processo de orientação profissional sobre a 
avaliação das afirmações pelos participantes foram repetidos os mesmos procedimentos realizados no pré-teste, igualmente sem qualquer feedback da pesquisadora sobre as respostas dos jovens.

Fase 5. Avaliação Final. O objetivo dessa fase era verificar se o processo de orientação profissional teve algum efeito sobre a maneira como os participantes avaliavam Nesta fase, o repertório dos participantes foi novamente avaliado pela Escala de Afirmações Profissionais com as 40 frases selecionadas pelos psicólogos clínicos na fase anterior.

\section{Análise dos dados}

Foram comparados os escores de pré e pós-teste das relações $\mathrm{AC} / \mathrm{CA}$ dos participantes, bem como os resultados dos participantes na Escala de Afirmações Profissionais na caracterização inicial de repertório e na avaliação final por meio do teste Wilcoxon para amostras pareadas. O nível de significância adotado para os testes foi de $5 \%$.

\section{Resultados}

A Figura 2 apresenta a porcentagem média de escolha dos estímulos verdadeiro (A1), falso (A2) e neutro para as afirmações no pré e pós-teste $\mathrm{CA}$ e $\mathrm{AC}$. A porção superior da Figura 2 resume os dados obtidos no pré-teste e a porção inferior, dados obtidos no pós teste.

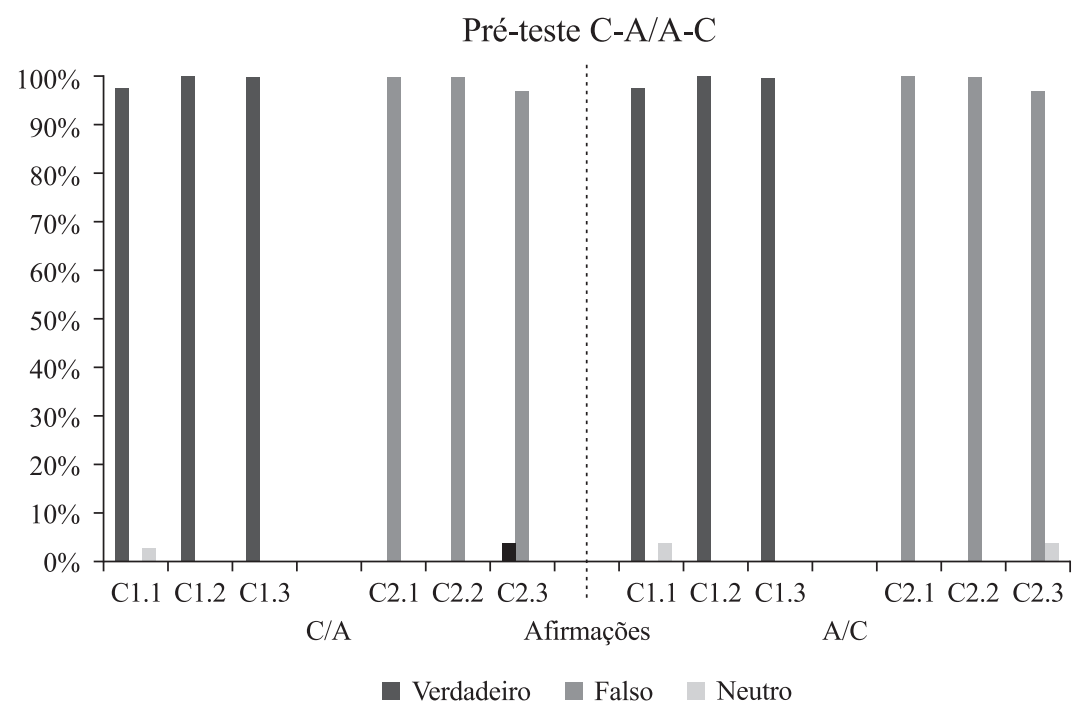

Pós-teste C-A/A-C

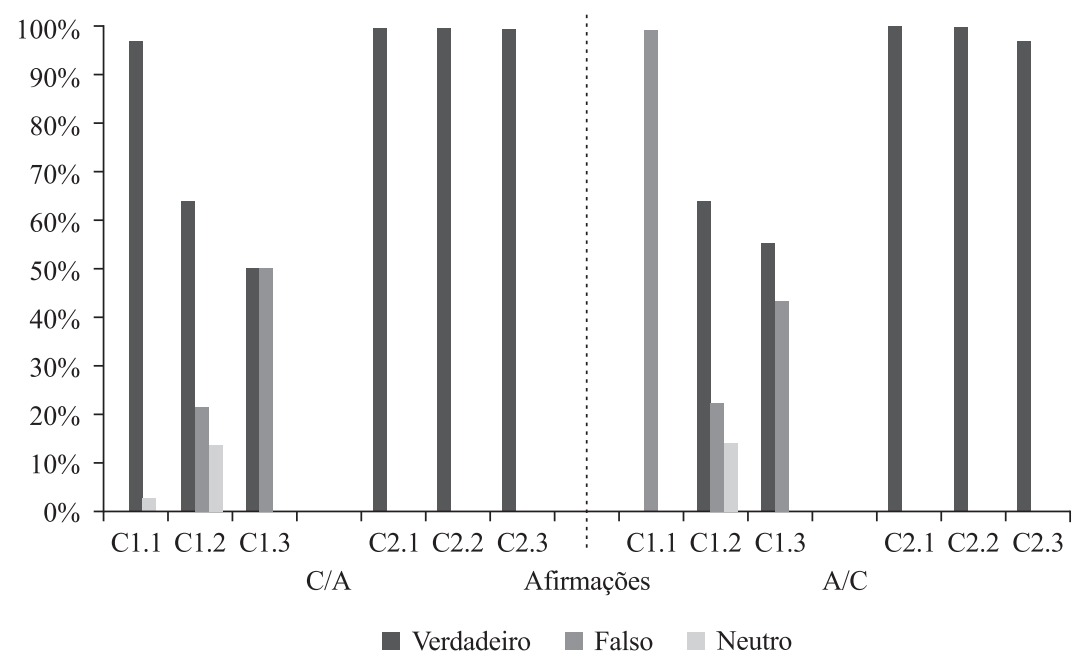

Figura 2. Porcentagem média de escolha dos estímulos verdadeiro (A1), falso (A2) e neutro pelo grupo para cada afirmação 
No pré-teste as afirmações $\mathrm{C} 1.1, \mathrm{C} 1.2$ e $\mathrm{C} 1.3$ foram consideradas verdadeiras em quase $100 \%$ das vezes e as afirmações C2.1, C2.2, C2.3 foram avaliadas como falsas em quase $100 \%$ das vezes. No pós-teste, observa-se que as afirmações consideradas previamente como falsas $(\mathrm{C} 2.1, \mathrm{C} 2.2, \mathrm{C} 2.3)$ passaram a ser consideradas predominantemente verdadeiras. Em relação às afirmações consideradas previamente verdadeiras, observou-se resultados variáveis: a afirmação $\mathrm{C} 1.2$ passou a ser avaliada como falsa em (97\%), C1.2 continuou sendo avaliada como verdadeira em $64 \%$ das escolhas (e $22 \%$ como falsa) e a afirmação C1.3 foi avaliada como verdadeira em 50\% das escolhas e como falsa das demais $50 \%$.

$\mathrm{O}$ teste Wilcoxon indicou diferenças significativas entre pré e pós-teste tanto em $\mathrm{AC}$ quanto em CA para as afirmações $\mathrm{C} 1.1(p=0,023), \mathrm{C} 2.1(p=0,014), \mathrm{C} 2.2$ $(p=0,020)$ e $\mathrm{C} 2.3(p=0,020)$, mas não para as afirmações C1.2 ( $p=0,157)$ e C1.3 ( $p=0,102)$, em nenhum dos testes. Não foram identificadas diferenças significativas entre pré e pós-teste para a porcentagem de escolhas da alternativa "neutro" C1.1 $(p=0,564), \mathrm{C} 1.2(p=0,317)$, $\mathrm{C} 1.3(p=1,0), \mathrm{C} 2.1(p=1,0), \mathrm{C} 2.2(p=0,317), \mathrm{C} 2.3$ $(p=1,0)$. Observou-se, portanto, uma inversão de avaliação de quatro das seis afirmativas, mas a manutenção das avaliações para as afirmações "Dinheiro é o mais importante na profissão" (C1.2) e "Apenas mulheres trabalham com profissões relacionadas às crianças" (C1.3).

Esses resultados foram analisados de acordo com os resultados dos participantes na Escala de Rigidez (Tabela 2) e no contexto geral das afirmações na Escala de Afirmações Profissionais (Figura 3), antes e após o programa de orientação profissional.

Escala de Afirmações Profissionais

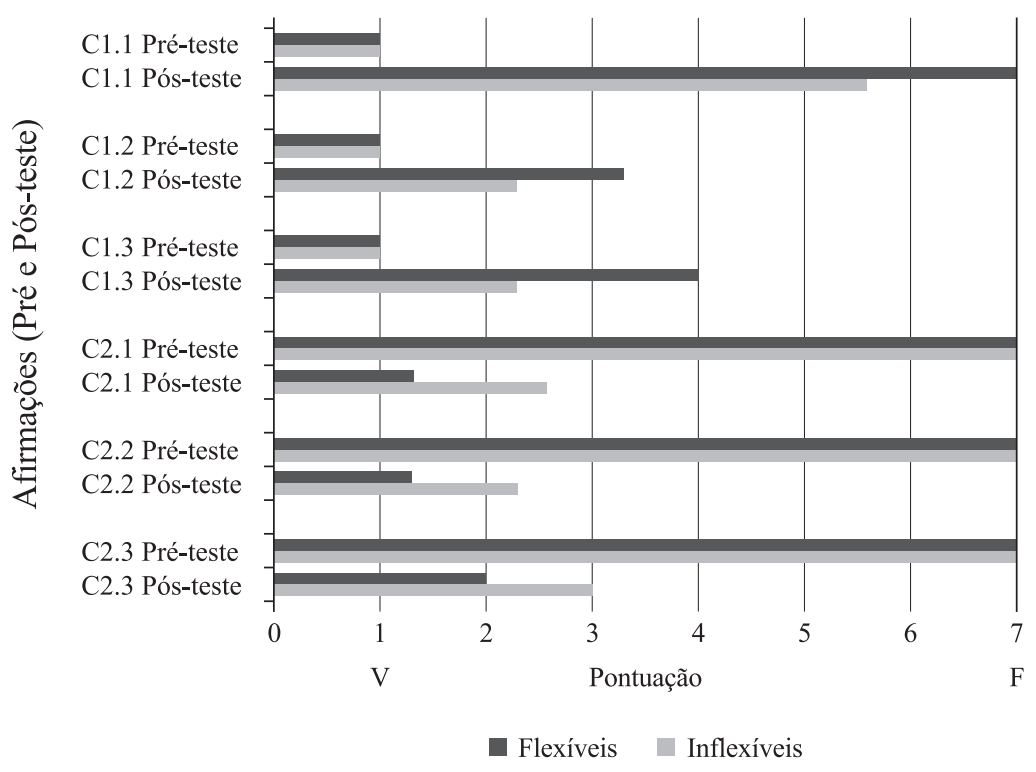

Figura 3. Média no pré e pós-teste na Escala de Afirmações

Profissionais de participantes flexíveis e inflexíveis

Na Figura 3 observa-se que as afirmações consideradas inicialmente verdadeiras foram avaliadas na escala como 1, indicando a pontuação máxima próxima ao verdadeiro. Após o procedimento de orientação profissional, as pontuações para as afirmações $\mathrm{C} 1.1, \mathrm{C} 1.2, \mathrm{C} 1.3$, nesta ordem, aumentaram para 7; 3,3 e 4 para participantes considerados flexíveis e 5,6; 2,3 e 2,3 para os inflexíveis. No caso das afirmações consideradas previamente como falsas, no início do procedimento foram pontuadas com o valor máximo (7), pontuação que diminuiu após o procedimento de $\mathrm{OP}$, chegando próximo à pontuação 1 (verdadeiro). Os participantes flexíveis consideraram as afirmações C2.1, C2.2 e C2.3, nesta ordem, com uma pontuação média no pós-teste de 1,$3 ; 1,3$ e 2 e para os inflexíveis 2,6;2,3 e 3.

O teste de Wilcoxon pareado indicou que os participantes aumentaram a pontuação do pré para o pós-teste diante das afirmações C.1.1 $(p=0,03)$, C1.2 $(p=0,03)$ e C1.3 $(p=0,03)$, e diminuíram do pré para o pós-teste para as afirmações C2.1 ( $p=0,03), \mathrm{C} 2 \cdot 2(p=0,03)$ e C2.3 $(p=0,02)$. 
Tabela 2

Resultado da Escala de Rigidez e porcentagem de mudança na avaliação das afirmações

\begin{tabular}{cccccccc}
\hline \multicolumn{7}{c}{ Porcentagem de mudança na avaliação } \\
\hline Participantes & $\begin{array}{c}\text { Teste de } \\
\text { flexibilidade }\end{array}$ & $\mathrm{C} 1.1$ & $\mathrm{C} 1.2$ & $\mathrm{C} 1.3$ & $\mathrm{C} 2.1$ & $\mathrm{C} 2.2$ & $\mathrm{C} 2.3$ \\
\hline P1 & Flexível & $100 \%$ & $83 \%$ & $100 \%$ & $100 \%$ & $100 \%$ & $100 \%$ \\
P2 & Flexível & $100 \%$ & $0 \%$ & $0 \%$ & $100 \%$ & $100 \%$ & $100 \%$ \\
P3 & Flexível & $100 \%$ & $100 \%$ & $100 \%$ & $100 \%$ & $100 \%$ & $100 \%$ \\
P4 & Inflexível & $100 \%$ & $0 \%$ & $0 \%$ & $100 \%$ & $100 \%$ & $100 \%$ \\
P5 & Inflexível & $100 \%$ & $0 \%$ & $0 \%$ & $100 \%$ & $100 \%$ & $100 \%$ \\
P6 & Inflexível & $83 \%$ & $0 \%$ & $0 \%$ & $100 \%$ & $100 \%$ & $100 \%$ \\
\hline
\end{tabular}

A Tabela 2 apresenta os resultados de mudança de avaliação dos participantes sobre as afirmações em função de seus resultados na Escala de Flexibilidade. Considerou-se que houve alteração na avaliação das afirmações quando foi verificada $100 \%$ de mudança de avaliação nos testes $\mathrm{AC} / \mathrm{CA}$ ou quando os participantes escolhiam em apenas uma tentativa o adjetivo "verdadeiro" diante das afirmações consideradas previamente verdadeiras, ou "falso" diante das afirmações previamente falsas (83\%). Não houve nenhuma alteração quando os participantes se mantiveram fazendo as mesmas avaliações sobre as afirmativas, depois das sessões de orientação profissional.

Dois participantes (P1 e P3), avaliados como flexíveis, alteraram suas avaliações sobre as afirmações depois das sessões de orientação profissional. O participante P2, também flexível, não alterou sua avaliação diante das afirmações C1.2 e C1.3. Cabe ressaltar que, diante dessas afirmações, o participante teve $100 \%$ de escolha pelo estímulo neutro. Os demais participantes, considerados inflexíveis, tiveram um padrão de responder idêntico ao de P2.

\section{Discussão}

O estudo teve como objetivo verificar os efeitos de um processo de OP em possíveis flexibilizações nas avaliações de descrições profissionais culturalmente difundidas entre estudantes do ensino médio considerados flexíveis e inflexíveis, segundo a Escala de Rigidez de Rehfisch (1958). A medida empregada para verificar tal mudança foi uma avaliação inicial e final, feita pelos participantes, sobre um conjunto de informações sobre escolha profissional e profissões, sem que essa avaliação recebesse qualquer feedback da pesquisadora.

De modo geral, dois participantes considerados flexíveis mudaram sua avaliação sobre todas as afirmações apresentadas; o terceiro participante considerado flexível teve desempenho idêntico ao dos considerados inflexíveis, mantendo sua avaliação sobre duas das seis afirmações (C1.2 e C1.3). Apesar de o desempenho de P2 e dos participantes considerados inflexíveis ser semelhante, P2 tendeu a relacionar essas afirmações ao estímulo neutro (e não ao estímulo verdadeiro, como no pré-teste) o que indica, ainda que em menor grau, uma mudança nas relações previamente estabelecidas. O mesmo não aconteceu com os participantes considerados inflexíveis.

Nesse sentido, o fator flexibilidade parece ser uma variável de interferência na avaliação das afirmações apresentadas, como mostram outros estudos sobre temas semelhantes (e.g., Oliveira \& Albuquerque, 2007; Paracampo, Souza, Matos, \& Albuquerque, 2001; Wulfert, Farkas, Hayes, \& Dougher, 1994). Uma contribuição desta pesquisa, portanto, foi identificar que a probabilidade do indivíduo mudar sua avaliação sobre afirmações acerca da escolha profissional também foi influenciada pela história prévia, que deve ser levada em conta. Embora a escala seja apenas uma medida da descrição verbal dos participantes, o estudo encontrou diferenças significativas de desempenho nos pré e pós-testes entre os flexíveis e inflexíveis, especialmente sobre as afirmações que têm um forte apelo cultural.

De fato, observou-se que algumas afirmações foram mais fáceis de serem reavaliadas pelos participantes que outras. As afirmações consideradas inicialmente falsas (C2.1, C2.2 e C2.3) foram avaliadas de forma diferente no pós-teste por todos os participantes, diferentemente do outro conjunto de afirmações, avaliadas inicialmente como verdadeiras. Isso pode ter ocorrido porque que a associação entre as afirmações apresentadas e seu valor (verdadeiro ou falso) poderia ser mais ou menos forte antes do experimento. É possível, por exemplo, que a afirmação C1.1 "sou livre para escolher o que quiser", apesar de ter sido considerada inicialmente verdadeira pelos participantes, não tivesse um "significado" de "correto" tão forte como aquele referente à afirmação C1.2 "Dinheiro é o mais importante na profissão". Estudos experimentais 
(Bortoloti \& de Rose, 2009, 2011; Bortoloti, Rodrigues, Cortez, Pimentel, \& de Rose, 2013) já demonstraram que há diferenças no grau de associação entre estímulos, mesmo entre aqueles pertencentes à mesma classe. Seguindo a lógica de Bortoloti e colaboradores, talvez algumas das afirmações consideradas inicialmente como verdadeiras neste estudo fossem "mais verdadeiras" que outras, o que não poderia ter sido verificado por conta do tipo de pré-teste realizado.

Analisando o conteúdo das afirmações C1.2 e C1.3 ("Dinheiro é o mais importante na profissão" e "Apenas mulheres trabalham com profissões relacionadas às crianças"), observa-se que estas parecem ser socialmente mais carregadas de significado, principalmente ao se considerar a condição socioeconômica dos participantes e do país, no caso da afirmação C1.2, e o significado social existente entre gênero e escolha profissional, no caso do estímulo C1.3 (ver De Carvalho \& De Rose, 2014; Mizael, de Almeida, Silveira, \& de Rose, 2016; Moxon, Keenan, \& Hine, 1993).

Essa diferença da avaliação das afirmações também foi observada na Escala de Afirmações Profissionais para as afirmações que foram alvo do procedimento. Depois do procedimento, houve modificação na avaliação das afirmações. Para os participantes que mudaram sua avaliação, houve uma modificação do polo de escolha (de verdadeiro para falso, e vice-versa). Com os participantes que não mudaram sua valência de escolha diante das afirmações $\mathrm{C} 1.2 \mathrm{e}$ C1.3, mesmo com uma menor flexibilização da avaliação, os seus escores ficaram na porção próxima à central.

Assim, os resultados permitem afirmar que o procedimento adotado propiciou maior flexibilidade na maneira como os estudantes avaliaram algumas afirmações potencialmente influenciadoras do processo de escolha profissional, podendo facilitar, inclusive, a escolha profissional dos estudantes. No processo de tomada de decisão profissional, certas descrições de contingências socialmente aceitas e não necessariamente relacionadas a contingências concretas sobre as profissões, como gênero, liberdade de escolha, mercado de trabalho etc. podem interferir de modo deletério na escolha profissional, dificultando o contato do sujeito com as contingências produzidas pelo seu próprio comportamento e fazendo com que ele fique mais sob controle dessas regras sociais. O papel do psicólogo e da orientação profissional é essencial aqui, uma vez que o processo de orientação envolve a aprendizagem e discriminação de autorreforçadores (interesses e habilidades), aspectos sobre a realidade profissional, planejamento de consequências futuras e tomada de decisão (Moura \& Menezes, 2004). A decisão é auxiliada quando, por exemplo, o orientando não conhece as habilidades exigidas por determinada profissão, o mercado de trabalho, as áreas de trabalho e, a partir do conhecimento e análise destas, tais variáveis alteram o valor reforçador de alguma opção em questão.

Durante as sessões de orientação profissional foram realizadas atividades para a discriminação de características pessoais, discriminação de reforçadores atuais e potenciais (aqueles que têm grande probabilidade de vir a controlar o comportamento futuro), informações sobre profissão, mercado de trabalho, entre outros fatores. Com esses procedimentos, os participantes puderam ampliar seu repertório de autoconhecimento, no sentido de reconhecer valores pessoais, sentimentos em relação à escolha profissional e seus planos futuros, obter informações profissionais e mercado de trabalho. Assim, ao enfocar outros importantes conjuntos de variáveis (profissões, cursos, mercado de trabalho, interesses, habilidades), a intervenção parece ter favorecido a diminuição do controle das afirmações preestabelecidas por agentes externos de controle, e isso aumentou a probabilidade dos participantes reavaliarem descrições de contingências pré-estabelecidas. Com esse aumento de repertório com informação sobre as questões profissionais e pessoais dos adolescentes, estes se tornam mais aptos a rever regras sociais e flexibilizar suas escolhas, ajudando a levantar possíveis alternativas sobre a indecisão.

\section{Considerações finais}

Entender como determinadas descrições de contingências são avaliadas como verdadeiras ou falsas, de que forma elas podem vir a controlar o comportamento dos indivíduos e como as avaliações sobre elas podem ser alteradas é fundamental para aqueles que estão preocupados em trabalhar com orientação profissional. $\mathrm{O}$ trabalho nessa área é entendido como um processo que promove melhoria na capacidade de discriminação e análise das variáveis envolvidas no processo de tomada de decisão, inclusive. O presente estudo contribuiu mostrando como o processo de orientação profissional pode desempenhar um papel importante na flexibilização da forma como o orientando avalia descrições socialmente difundidas sobre escolha profissional e sobre as diferentes carreiras.

É importante ponderar, no entanto, que é necessário cautela na generalização dos resultados obtidos em situação experimental para o ambiente natural, especialmente em função do número reduzido de participantes. Especula-se que a aparente inflexibilidade observada na situação experimental seja um padrão mais extenso do comportamento verbal e manifesto em ambiente natural, 
mas os dados obtidos com a presente pesquisa não sustentam empiricamente tais conclusões. É importante replicar os procedimentos em futuros estudos com amostras maiores.

\section{Referências}

Albuquerque, L. C. (2001). Definições de regras. Em H. J. Guilhardi, M. B. B. P. Madi, P. P. Queiroz, \& M. C. Scoz (Orgs.). Sobre comportamento e cognição: Expondo a variabilidade (pp.132-140). Santo André: ARBytes.

Albuquerque, L. C., Souza, D. G., Matos, M. A., \& Paracampo, C. C. P. (2003). Análise dos efeitos de histórias experimentais sobre o seguimento subsequente de regras. Acta Comportamentalia, 11, 87-126. Recuperado em 06 maio 2017, de http://www.revistas.unam.mx/index.php/acom/article/view/14614

Albuquerque, L. C. (2005). Regras como instrumento de análise do comportamento. In L. C. Albuquerque (Ed.), Estudos do comportamento (pp.143-176). Belém, PA: Editora da Universidade Federal do Pará.

Albuquerque, L. C., \& Silva, F. M. (2006). Efeitos da exposição a mudanças nas contingências sobre o seguir regras. Psicologia: Teoria e Pesquisa, 22, 101-112. DOI: http://dx.doi.org/10.1590/S0102-37722006000100013

Albuquerque, L. C., Reis, A. A., \& Paracampo, C. C. P. (2006). Efeitos de uma história de reforço contínuo sobre o seguimento de regras. Acta Comportamentalia, 14, 47-75. Recuperado em 06 maio 2017, de http://pepsic.bvsalud. org/pdf/actac/v14n1/v14n1a03.pdf

Albuquerque, L. C., \& Paracampo, C. C. P. (2010). Análise do controle por regras. Psicologia USP, 21, 253-273. DOI: http://dx.doi.org/10.1590/S0103-65642010000200004

Albuquerque, L. C., Paracampo, C. C. P., \& Allan, S. (2017). Análise dos efeitos de variáveis históricas sobre o seguimento de regra discrepante. Acta Comportamentalia: Revista Latina de Análisis de Comportamiento. Recuperado em 06 maio 2017, de http://www.redalyc.org/articulo.oa?id=274553648002

Albuquerque, L. C., Silva, F. M., \& Paracampo, C. C.P. (2014). Análise de variáveis que podem interferir no comportamento de seguir regras discrepantes. Acta Comportamentalia, 22, 51-71. Recuperado em 06 maio 2017, de http://pepsic. bvsalud.org/pdf/actac/v22n1/a05.pdf

Almeida, F. H., Melo- Silva, L. L., \& Santos, M. A. (2017). Grupo operativo com pais de jovens em processo de escolha da carreira. Revista da spagesp, v. 18, p. 80-100.

Bandura, A. (1979). Modificação do Comportamento. Rio de Janeiro: Interamericana.

Bortoloti, R., \& de Rose, J. C. (2009). Assessment of the relatedness of equivalent stimuli through a semantic differential. The Psychological Record, 59, 563-590. Recuperado em 06 maio 2017, de http://opensiuc.lib.siu.edu/tpr/vol59/ iss $4 / 5 /$

Bortoloti, R., \& de Rose, J. C. (2011). “Orwellian” account of stimulus equivalence. Are some stimuli more equivalent than others?. European Journal of Behavior Analysis, v. 12, p. 121-134. DOI: https://doi.org/10.1080/15021149.20 11.11434359

Bortoloti, R., Rodrigues, N. C., Cortez, M. D., Pimentel, N., \& Rose, J. C. de. (2013). Overtraining increases the strength of equivalence relations. Psychology \& Neuroscience, 6(3), 357-364. DOI: https://dx.doi.org/10.3922/j.psns.2013.3.13

Catania, A. C. (1998). Learning (4th ed.). Upper Saddle River, NJ: Prentice-Hall.

Catania, A. C., Shimoff, E., \& Matthews, B. A. (1989). An experimental analysis of rule-governed behavior. In S. C. Hayes (Org.), Rule-governed behavior: Cognition, contingencies, and instructional control (pp. 119-150). New York: Plenum.

Catania, A. C., Shimoff, E., \& Matthews, A. (1990).An experimental analysis of rule-governed behavior. In S. C. Hayes (Org.), Rule-governed behavior: Cognition, contingencies, and instructional control (pp.119-150). New York: Plenum.

Catania, A. C. (1999). Aprendizagem: Comportamento, linguagem e cognição. Porto Alegre: Artmed.

Cerutti, D. T. (1989). Discrimination theory of rule-governed behavior. Journal of the Experimental Analysis of Behavior, 51, 259-276. DOI: 10.1901/jeab.1989.51-259

De Carvalho, M. P., De Rose, J. C. (2014). Understanding Racial Attitudes through the Stimulus Equivalence Paradigm. The Psychological Record, v. 64, p. 527-536, 2014. DOI: https://doi.org/10.1007/s40732-014-0049-4

Jonas, A. (2001). Efeitos de instruções sobre o desempenho em matching to sample e sua relação com padrões de sensibilidade comportamental a contingências. Tese de doutorado não-publicada, Instituto de Psicologia, Universidade de São Paulo. São Paulo, SP. 
Joyce, J. H., \& Chase, P. N. (1990). Effects of response variability on the sensitivity of rule governed behavior. Journal of the Experimental Analysis of Behavior, 54, 251-262. DOI: 10.1901/jeab.1990.54-251

Kravas, K. J. (1973). Reducing levels of dogmatism and rigidity among educational helpers. Journal of the Student Personnel Association for Teacher Education, 12, 38-43. DOI: 10.1002/j.2164-6171.1973.tb00044.x

Krumboltz, J. D, \& Worthington, R. L. (1999). The school-to-work transition from a learning theory perspective. The career Development Quarterly, 47, 312-325. DOI: 10.1002/j.2161-0045.1999.tb00740.x

Krumboltz, J. D. (1992). The Wisdom of indecision. Journal of Vocational Behavior, 41, 239-244. DOI: https://doi. org/10.1016/0001-8791(92)90025-U

Krumboltz, J. D. (1998). Serendipity is not serendipitous. Journal of Counseling Psychology, 45, 390-392. DOI: http:// dx.doi.org/10.1037/0022-0167.45.4.390

Le François, J. R., Chase, P. N., \& Joyce, J. (1988). The effects of a variety of instructions on human fixed interval performance. Journal of the Experimental Analysis of Behavior, 49, 383-393. DOI: 10.1901/jeab.1988.49-383

Leal, M. S., Melo- Silva, L. L., \& Teixeira, M. O. (2015). Crenças para lidar com tarefas de carreira em estudantes do ensino médio. Avaliação Psicológica (Online), v. 14, p. 125-132, 2015. DOI: 10.15689/ap.2015.1401.14

Lent, R. W., Brown, S. D., \& Hackett, G. (2000). Contextual supports and barriers to career choice: A Social cognitive analysis. Journal of Counseling Psychology, 47, 36-49. DOI: http://dx.doi.org/10.1037/0022-0167.47.1.36

Levenfus, R. S., \& Soares, D. H. P. (2002). Orientação Vocacional Ocupacional: Novos achados teóricos, técnicos e instrumentais para a clínica, a escola e a empresa. Porto Alegre: Artmed.

Lima, F. M., Albuquerque, L. C., \& Paracampo, C. C. P. (2017). Effects of Listener's Histories on Following Rules that are Discrepant to Contingencies. Temas em Psicologia, 25, 4, 1959-1975. DOI: 10.9788/TP2017.4-21En

Linn, B. S., Moravec, J., \& Zeppa, R. (1982). The impact of clinical experience on attitudes of junior medical students about death and dying. Journal of Medical Education, 57, 684-691.

Manaia, M., Leal, M. S., Shimada, M., \& Melo-Silva, L. L. (2014). Instrumentos de avaliação psicológica na intervenção em Orientação Profissional: estudo de caso. In: S. R. Pasian; E. T. K. Okino; D. M. Amparo; F. de L. Osório; F. R. Freitas; S. R. Loureiro. (Org.). Desafios para a prática ética da Avaliação Psicológica. 1ed. Ribeirão Preto, São Paulo: ASBRo.

Martins, C. R. (1978). Psicologia do Comportamento Vocacional. São Paulo, SP: EPU.

Melo-Silva, L. L. (2011). Intervenção e avaliação em orientação profissional e de carreira. In M. A. Ribeiro \& L. L. Melo-Silva (Orgs.). Enfoques teóricos contemporâneos e modelos de intervenção. Compêndio de orientação profissional e de carreira (Vol. 2, pp. 155-192). São Paulo: Vetor.

Melo- Silva, L. L., \& Alonso, W. C. (2013). Avaliação de uma intervenção em orientação profissional na perspectiva de ex-estagiários. Psicologia: Ciência e Profissão (Impresso), v. 33, p. 84-99.

Mitchell, L. K., \& Krumboltz, J. D. (1996). Krumboltz's learning theory of career choice and counseling. In D. Brown, \& L. Brooks (Eds.), Career choice and development (3rd ed., pp. 233-280). San Francisco: Jossey-Bass.

Mitchell, K. E., Levin, A. S., \& Krumboltz, J. D. (1999). Planned happenstance: Constructing unexpected career opportunities. Journal of Counseling e Development, 77, 115-124. DOI: 10.1002/j.1556-6676.1999.tb02431.x

Mizael, T. M., De Almeida, J. H., Silveira, C. C., \& De Rose, J. C. (2016). Changing Racial Bias by Transfer of Functions in Equivalence Classes. The Psychological Record, v. 66, p. 1-12. DOI: https://doi.org/10.1007/ s40732-016-0185-0

Moraes, C. M. P., \& Lima, A. O. (2014). Vínculo paterno y elección profesional del adolescente. Alternativas em Psicología, 17(29), 74-89. Recuperado em 06 maio 2017, de http://www.alternativas.me/index.php/ num29/46-6-vinculo-paterno-y- leccionprofesional-del-adolescente.

Moura, C. B, Sampaio, A. C. P., Gemelli, K. R., Rodrigues, L. D., \& Menezes, M. V. (2005). Avaliação de um programa comportamental de orientação profissional para adolescentes. Revista Brasileira de Orientação Profissional, 6(1), 25-40. Recuperado em 06 maio 2017, de http://pepsic.bvsalud.org/pdf/rbop/v6n1/v6n1a04.pdf

Moura, C. B, Sampaio, A. C. P., Menezes, M. V., \& Rodrigues, L. D. (2003). Uso de relatos escritos como instrumento de avaliação e intervenção em orientação profissional. Estudos de Psicologia, 20(3), 83-98. DOI: http://dx.doi. org/10.1590/S0103-166X2003000300007

Moura, C. B., \& Menezes, M. V. (2004). Mudando de opinião: Análise de um grupo de pessoas em condição de re-escolha profissional. Revista Brasileira de Orientação Profissional, 5(1), 29-45. Recuperado em 06 maio 2017, de http:// pepsic.bvsalud.org/pdf/rbop/v5n1/v5n1a04.pdf 
Moura, C. B., \& Silveira, J. M. (2002). Orientação Vocacional sob o enfoque da Análise do comportamento: Avaliação de uma experiência. Estudos de Psicologia (Campinas), 19(1), 5-14. DOI: https://dx.doi.org/10.1590/ S0103-166X2002000100001

Moura, C. B. (2011). Orientação Profissional sob o enfoque da análise do comportamento ( $3^{\mathrm{a}}$ ed.). Campinas: Alínea.

Moxon, P. D., Keenan, M., \& Hine, L. (1993). Gender hole stereotyping and stimulus equivalence. The Psychological Record, 43, 381-394.

Naftulin, D. H., Donnelly, F. A., \& Wolkon, G. H. (1974). Selection of nuns for training as mental health counselors. Journal of Community Psychology, 2, 366-369. DOI: 10.1002/1520-6629(197410)2:4<366::AIDJCOP2290020409>3.0.CO;2-L

Oliveira, C. T., \& Dias, A. C. G. (2013). Percepções parentais sobre sua participação no desenvolvimento profissional dos filhos universitários. Revista Brasileira de Orientação Profissional, 14(1), 61-72. Recuperado em 06 maio 2017, de http://pepsic.bvsalud.org/pdf/rbop/v14n1/07.pdf

Oliveira, M. C., Silva, B. R., Garcia, R. G., Melo-Silva, L. L., \& Teixeira, M. A. P. (2014). Escala de autoeficácia no aconselhamento de carreira: adaptação e evidências de validade. Avaliação Psicológica, 13, 371-381. Recuperado em 06 maio 2017, de http://pepsic.bvsalud.org/pdf/avp/v13n3/v13n3a09.pdf

Oliveira, V. L., \& Albuquerque, L. C. (2007). Efeitos de histórias experimentais e de esquemas de reforço sobre o seguir regras. Psicologia: Teoria e Pesquisa, 23, 217-228. DOI: http://dx.doi.org/10.1590/S0102-37722007000200012

Orlando, A. F. (2009). Uma infra-estrutura computacional para o gerenciamento de programas de ensino individualizado. Dissertação. Mestrado em Ciência da Computação - UFSCar.

Paracampo, C. C. P., \& Albuquerque, L. C. (2004). Análise do papel das consequências programadas no seguimento de regras. Interação em Psicologia, 8, 237-245. DOI: http://dx.doi.org/10.5380/psi.v8i2.3259

Paracampo, C. C. P., de Souza, D. G., Matos, M. A., \& Albuquerque, L. C. (2001). Efeitos de mudança em contingências de reforço sobre o comportamento verbal e não verbal. Acta Comportamentalia, 9, 31-55. Recuperado em 06 maio 2017, de http://www.journals.unam.mx/index.php/acom/article/view/14632

Paracampo, C. C. P., Souza, L. M., \& Albuquerque, L. C. (2014). Variáveis que podem interferir no seguir regras de participantes flexíveis e inflexíveis. Psicologia: Reflexão e Crítica, 27(1), 124-133. DOI: https://dx.doi.org/10.1590/ S0102-79722014000100014

Rehfisch, J. M. (1958). A Scale for Personality rigidity. Journal of Consulting Psychology, 1, 11-15. DOI: http://dx.doi. org $/ 10.1037 / \mathrm{h} 0047945$

Ribeiro, M. A. (2011). Orientação profissional: uma proposta de guia terminológico. In M. A. Ribeiro \& L. L. Melo-Silva (Orgs.), Compêndio de Orientação Profissional e de Carreira (pp. 23-66). São Paulo: Vetor.

Ribeiro, M. A., \& Uvaldo, M. C. C. (2007). Frank Parsons: Trajetória do pioneiro da orientação vocacional, profissional e de carreira. Revista Brasileira de Orientação Profissional, 8(1), 19-31. Recuperado em 06 maio 2017, de http:// pepsic.bvsalud.org/pdf/rbop/v8n1/v8n1a03.pdf

Sampson Jr, J. P., Peterson, G. W., Lenz, J. G, \& Reardon, R. C. (1992). A cognitive approach to career services: Translating concepts into practice. The career Development Quarterly, 41, 67-74. DOI: 10.1002/j.2161-0045.1992.tb00360.x

Santos, J. G. W. Paracampo, C. C. P., \& Albuquerque, L. C. (2004). Analise dos efeitos de histórias de variação comportamental sobre o seguimento de regras. Psicologia, Reflexão e Crítica, 17(3), 413-425. DOI: http://dx.doi. org/10.1590/S0102-79722004000300013

Sawitri, D. R., Creed, P. A., \& Zimmer-Gemback, M. J. (2014). Parental influences and adolescent career behaviours in a collectivist cultural setting. International Journal for Educational and Vocational Guidance, 14(2), 161-180. DOI: https://doi.org/10.1007/s10775-013-9247-x

Schaie K. W., Dutta R., \& Willis S. L. (1991). The relationship between rigidity-flexibility and cognitive abilities in adulthood. Psychology and Aging, 6, 371-383. DOI: http://dx.doi.org/10.1037/0882-7974.6.3.371

Sharf, R. S. (1992). Applying career development theory to counseling. California: Brooks/Cole.

Silva, B. R., Oliveira, M. C., \& Melo-Silva, L. L. (2014). Autoeficácia no aconselhamento de carreira: Estudo com orientadores profissionais brasileiros. Revista Brasileira de Orientação Profissional, 15(1), 5-13. Recuperado em 06 maio 2017, de http://pepsic.bvsalud.org/pdf/rbop/v15n1/03.pdf

Skinner, B. F. (1969). Contingencies of reinforcement: A theoretical analysis. New York: Appleton-Century-Crofts.

Skinner, B. F. (1989). Ciência e comportamento humano. São Paulo: Martins Fontes. Tradução realizada por J. C. Todorov \& R. Azzi. São Paulo: Martins Fontes. (trabalho original publicado em 1953). 
Swanson, J. L., \& Gore Jr., P. A. (2000). Advances in vocational psychology theory and research. In S. D. Brown \& R. W. Lent (Org.), Handbook of counseling psychology (pp. 233-269). New York: Wiley.

Teixeira, M. A. P., Lassance, M. C. P., \& Melo-Silva, L. L.. (2012). Síntese das discussões e propostas do Grupo de Trabalho: Avaliação psicológica no domínio da orientação profissional e de carreira. Revista Brasileira de Orientação Profissional, 13(1), 121-124. Recuperado em 06 maio 2017, de http://pepsic.bvsalud.org/pdf/rbop/v13n1/14.pdf

Torgrud, L. J., \& Holborn, S.W. (1990).The effects of verbal performance descriptions on nonverbal operant responding. Journal of the Experimental Analysis of Behavior, 54(3), 273-291. DOI: 10.1901/jeab.1990.54-273

Vollhardt, L. (1990). Rigidity: A comparison by age and gender. Social Behavior and Personality: An international Journal, 18(1), 17-26. DOI: https://doi.org/10.2224/sbp.1990.18.1.17

Wulfert, E., Greenway, D. E., Farkas, P., Hayes, S. C., \& Dougher, M. J. (1994). Correlation between a personality test for rigidity and rule-governed insensitivity to operant contingencies. Journal of Applied Behavior Analysis, 27(4), 659-671. DOI: 10.1901/jaba.1994.27-659

Zettle, R. D., \& Hayes, S. C. (1982). Rule governed behavior: A potential theorical framework for cognitive behavior therapy. In P. C. Kendall (Ed.), Advances in cognitive behavioral research and therapy (pp. 191-218). New York: Academic Press.

Recebido: 06/05/2017

$1^{a}$ reformulação: 09/10/2017

Aceite final: 20/12/2017

Sobre as autoras

Nathália Sabaine Cippola é Mestre e Doutora do programa de pós graduação em psicologia da Universidade Federal de São Carlos.

Camila Domeniconi é Doutora pela Universidade Federal de São Carlos, pós doutora pela Universidade do Minho e pela Universidad de Barcelona. Pesquisadora do Instituto Nacional de Ciência e Tecnologia sobre Comportamento, Cognição e Ensino (INCT - ECCE). Docente do departamento de Psicologia da Universidade Federal de São Carlos.

Andréia Schmidt é Doutora em Psicologia (Psicologia Experimental) pela Universidade de São Paulo. Pesquisadora do Instituto Nacional de Ciência e Tecnologia sobre Comportamento, Cognição e Ensino (INCT - ECCE). Docente do curso de Psicologia da Universidade de São Paulo, campus Ribeirão Preto. 